\title{
Correction to: a Phase 2 Study

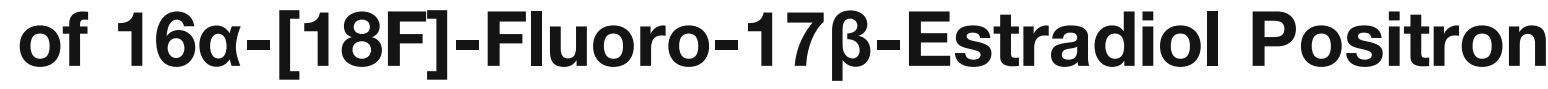 Emission Tomography (FES-PET) as a Marker of Hormone Sensitivity in Metastatic Breast Cancer (MBC)
}

Lanell M. Peterson, ${ }^{1}$ Brenda F. Kurland, ${ }^{2,3}$ Erin K. Schubert, ${ }^{1,4}$ Jeanne M. Link, ${ }^{5}$ V. K. Gadi, ${ }^{2,6}$ Jennifer M. Specht, ${ }^{2,6}$ Janet F. Eary, ${ }^{5}$ Peggy Porter, ${ }^{2}$ Lalitha K. Shankar, ${ }^{7}$ David A. Mankoff, ${ }^{1,4}$ Hannah M. Linden ${ }^{2,6}$

${ }^{1}$ Radiology/Nuclear Medicine, University of Washington Medical Center-Seattle Cancer Care Alliance, Seattle, WA, USA

${ }^{2}$ Clinical Research Division, Fred Hutchinson Cancer Research Center, Seattle, WA, USA

${ }^{3}$ Biostatistics, University of Pittsburgh, Pittsburgh, PA, USA

${ }^{4}$ Radiology/Nuclear Medicine, University of Pennsylvania, Philadelphia, PA, USA

${ }^{5}$ Radiology/Nuclear Medicine, University of Washington Medical Center, Seattle, WA, USA

${ }^{6}$ Medical Oncology, University of Washington Medical Center-Seattle Cancer Care Alliance, Seattle, WA, USA

${ }^{7}$ Cancer Imaging Program, National Cancer Institute, Bethesda, MD, USA

Correction to: Mol Imaging Biol https://doi.org/10.1007/s11307-013-0699-7

Two data points from Table 1. (continued) were published in error. The corrected data in Table 1. (continued) are shown, in italic.
The online version of the original article can be found at https://doi.org/ 10.1007/s11307-013-0699-7

Correspondence to: Lanell Peterson; e-mail: lanell@uw.edu
Table 1. (continued)

\begin{tabular}{ll}
\hline Patients $(n=19)$ & N (\%) or median (min-max) \\
\hline HER2neu (metastatic lesion) & \\
Positive & $3(20 \%)$ \\
Negative & $8(53 \%)$ \\
Unknown & $4(27 \%)$ \\
Clinical response & $4(21 \%)$ \\
Partial response & $4(21 \%)$ \\
Stable disease & $7(37 \%)$ \\
Radiographic progressive disease & $4(21 \%)$ \\
No response assessment ${ }^{\mathrm{c}}$ &
\end{tabular}

${ }^{\mathrm{a}}$ One patient also underwent ovarian suppression

${ }^{\mathrm{b}}$ When Allred scores were available, scoring was 0 "negative," 2-6 "weakly positive," 7-8 "positive." When Allred scores were not available, clinical report scores of $1+$ were "weakly positive"

'Includes two patients who did not undergo endocrine monotherapy, one who had complications from a non-breast cancer-related surgery after 2 days of anastrozole, and one who changed therapy (from tamoxifen) when a successful metastatic biopsy revealed ER- disease 